\title{
Mantle cell lymphoma of the prostate
}

\author{
Abuhjar Abdussalam, MBChB; Ronald G. Gerridzen, MD, FRCSC, FACS
}

\section{Abstract}

Lymphoma of the prostate, either primary or secondary, is very rare. We report the case of an 82-year-old man with symptoms of bladder outlet obstruction presumably due to benign prostate hyperplasia $(\mathrm{BPH})$. He underwent an uneventful transurethral resection of the prostate. Pathological review of the resected tissue demonstrated substantial infiltration by an atypical lymphoid infiltrate positive for CD20, BCL2, CD5, $\kappa$ light chain and cycline D1. Histology and immunoprofile were consistent with mantle cell lymphoma.

Can Urol Assoc J 2009;3(6):E94-E95

\section{Case report}

An 82-year-old man presented with increasing symptoms of bladder outlet obstruction. His medical history included repair of an abdominal aortic aneurysm in late 2005 followed by a brief period of urinary retention that spontaneously resolved. He also had hypercholesterolemia and diabetes. He had no family history of prostate cancer and his prostate-specific antigen (PSA) level was 2.4.

Cystoscopy demonstrated a moderately large prostate with a prominent middle lobe and a moderately trabeculated bladder with small diverticula. After medical therapy failed to improve his symptoms he underwent transurethral resection of the prostate. We resected $28 \mathrm{~g}$ of tissue with substantial clinical improvement. Microscopically, the tissue was substantially infiltrated by an atypical lymphoid infiltrate positive for CD20, BCL2, CD5 (weakly), $\kappa$ light chain and cycline D1, consistent with mantle cell lymphoma (Fig. 1 and Fig. 2).

A computed tomography (CT) scan of his abdomen and thorax showed marked lymphadenopathy consistent with stage-IIIA mantle cell lymphoma. He had no constitutional or systemic symptoms. We referred him to a medical oncologist, and he received $\mathrm{CHOP} /$ Rituxan chemotherapy.

\section{Discussion}

Lymphoma of the prostate, either primary or secondary, is very rare. Most cases are diagnosed at autopsy. A 20-year review of 1474 patients with prostate cancer was completed at the MD Anderson Cancer Center; only 18 nonleukemic secondary cancers were identified. ${ }^{1}$ An autopsy series of 6000 male patients who died of cancer revealed only 185 $(3.1 \%)$ with metastatic prostate cancer, of which only 49 $(0.8 \%)$ had non-Hodgkins lymphoma. ${ }^{2}$ According to the criteria of Bostwick and colleagues, ${ }^{2}$ primary prostatic lymphoma will be diagnosed only if the following criteria are fulfilled: primary symptoms are attributable to prostatic enlargement; the major bulk of disease is localized to the prostate; and lymph nodes, liver or spleen are not involved within 1 month of diagnosis. Therefore, even though our patient presented with symptoms attributable to prostatic enlargement, he should be classified as having secondary prostatic lymphoma. Only 2 cases of mantle cell lymphoma

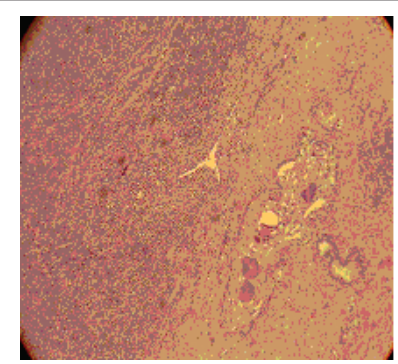

10x power, lymphoid infiltrate and adjacent acini

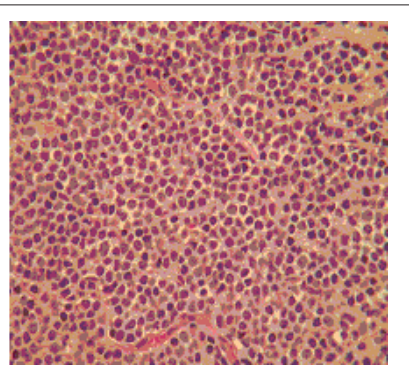

High power H\&E, lymphoma cells
Fig. 1. Prostatic tissue infiltrated by an atypical lymphoid infiltrate under low and high power.

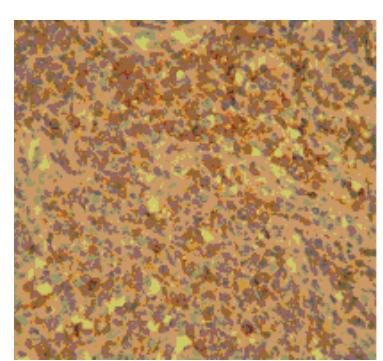

CD5, membranous positivity

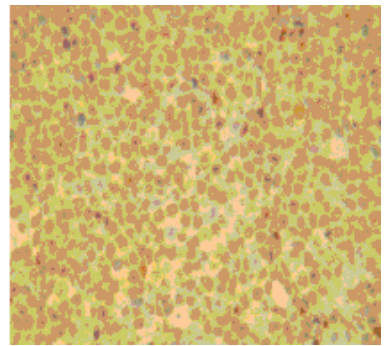

Cyclin D1, nuclear positivity
Fig. 2. Prostatic tissue infiltrated by an atypical lymphoid infiltrate positive for CD5 (weak), Kappa light chain and cycline D1. 
involving the prostate have been reported. The first ${ }^{3}$ was reported in December 2003 from the Queen Mary Hospital, University of Hong Kong, and the second ${ }^{4}$ in February 2006 from Cleveland.

\section{Conclusion}

Our patient had a highly unusual presentation of mantle cell lymphoma involving the prostate. There were no other systemic manifestations of the primary disease, therefore the pathological findings were unsuspected.

From the Division of Urology, Department of Surgery, University of Ottawa, Ottawa, Ont.

This paper has been peer-reviewed.

Competing interests: None declared.

\section{References}

1. Zein TA, Huben R, Lane W et al. Secondary tumors of the prostate. J. Urol 1985;133:615-6.

2. Bostwick DG, Iczkowski KA, Amin MB, et al. Malignant lymphoma involving the prostate: report of 62 cases. Cancer 1998;83:732-8.

3. Chim CS, Loong F, Yau T, et al. Common malignancies with uncommon sites of presentation case 2 : mantle cell lymphoma of the prostate gland. J Clin Oncol 2003;21:4456-8.

4. Genkin I. Mantle cell lymphoma of prostate. Commun Oncol 2006;3:69.

Correspondence: Dr. Ronald G. Gerridzen, The Ottawa Hospital - Civic Campus, Division of Urology, B327, 1053 Carling Ave., Ottawa ON KIY 4E9; rgerridzen@ottawahospital.on.ca 